\title{
新型哌啶噻唑类化合物的合成及杀虫活性
}

\author{
丁成荣潘亚运殷许谭成侠*张国富*
}

(浙江工业大学 杭州 310032)

\begin{abstract}
摘要 为了探寻新型的生物活性化合物, 设计并合成了 12 个未见文献报道的新型哌啶噻唑类衍生物. 生物活性测试研 究发现，在 $500 \mu \mathrm{g} / \mathrm{mL}$ 浓度下，目标化合物对粘虫表现出良好的抑制活性，而且在 $100 \mu \mathrm{g} / \mathrm{mL}$ 下，(4-(5-(3-氯苯基)-4-甲 基噻唑-2-基)哌啶-1-基)(4-甲基哌嗪-1-基)甲酮(1)、(5-(3-氯苯基)-4-甲基噻唑-2-基)哌啶-1-基)(4-硝基- $1 H$-吡唑-3-基)甲 酮(1g)对粘虫的抑制率均达 80\%以上，另外在 $20 \mu \mathrm{g} / \mathrm{mL}$ 下(5-(3-氯苯基)-4-甲基噻唑-2-基)哌啶-1-基)(4-硝基-1H-吡唑-3基)甲酮(1g)仍具有 $50 \%$ 的杀虫活性.
\end{abstract}

关键词 氟噻唑吡乙酮; 哌啶噻唑类衍生物; 杀虫活性

\section{Synthesis and Insecticidal Activity of Novel Piperidine Thiazole Compounds}

\author{
Ding, Chengrong Pan, Yayun Yin, Xu Tan, Chengxia* Zhang, Guofu* \\ (College of Chemical Engineering and Materials Science, Zhejiang University of Technology, Hangzhou 310032)
}

\begin{abstract}
Twelve new piperdine thiazole compounds were designed and synthesized in search of new bioactive compounds. The preliminary bioassay showed that at the concentration of $500 \mu \mathrm{g} / \mathrm{mL}$ the lethal rates of the target compounds possessed certain insceticidal activities against armyworm, and at the concentration of $100 \mu \mathrm{g} / \mathrm{mL}$ the lethal rates of (4-(5-(3-chlorophenyl)-4-methylthiazol-2-yl)piperidin-1-yl)(4-methylpiperazin-1-yl)methanone (1f) and (4-(5-(4-chlorophenyl)-4-methylthiazol-2-yl)piperidin-1-yl)nitro-1H-pyrazol-3-yl)methanone (1g) against armyworm were $80 \%$ and $100 \%$, respectively. Further screening at concentrations of $20 \mu \mathrm{g} / \mathrm{mL}$, the lethal rate of (4-(5-(4-chlorophenyl)-4-methylthiazol-2-yl)piperidin-1yl)(4-nitro-1 $H$-pyrazol- 3-yl)methanone (1g) against armyworm was 50\%.
\end{abstract}

Keywords oxathiapiprolin; piperidine-thiazolederivatives; insecticidal activity

哌啶噻唑类化合物 ${ }^{[13]}$ 是一类重要的药物中间体, 并且是微粒体甘油三酯转移蛋白(MTP)抑制剂 ${ }^{[4.5]}$. 在新 农药创制研究的过程中发现, 此类化合物具有一定的杀 菌活性 ${ }^{[6.7]}$, 是农药先导研究的热点. 杜邦公司在农药领 域成功开发出哌啶噻唑类农药商品: 增威赢绿(Zorvec Enicade). 该品种具有新型的杀菌作用位点，低毒、高效， 尤其对卵菌纲病原菌具有优异的杀菌活性. 近几年哌啶 噻唑类化合物得到了一定的发展, 对已报道的哌啶噻唑 类化合物分析发现(图 1), 该类化合物在结构上继承了 氟噻唑吡乙酮 ${ }^{[8 \sim 10]}$ 的骨架, 具有不同程度的杀菌活 性 ${ }^{[11 \sim 19]}$, 但在杀虫、除草方向的研究成果未见报道.

在研究已报道的哌啶噻唑类化合物的结构中发现, 哌啶与噻唑相连的结构未发生变动, 是一种新型的官能
团组，在对该类化合物结构优化实验研究中发现，结构 6 具有一定的杀虫活性, 因此本课题组在结构 6 的基础 上设计 ${ }^{[20 ~ 26]}$ 并合成了 12 个哌啶类噻唑类化合物(其合 成路线如 Scheme 1 所示), 所有目标化合物的结构经 ${ }^{1} \mathrm{H}$ NMR, ${ }^{13} \mathrm{C}$ NMR 和 HRMS 确认. 初步生物活性测试表 明，该类化合物具有良好的粘虫杀灭活性，具有继续深 入研究的必要.

\section{1 结果与讨论}

\section{1 化合物的合成及结构表征}

以间氯苯胺为底物, 经重氮化、氯化、环化得到中 间体 $\mathbf{5}$, 中间体 $\mathbf{5}$ 脱保护后与酰氯反应最终得到目标化 合物 $1 \mathrm{a} \sim$ II. 其中由中间体 2 和中间体 4 环化形成中

\footnotetext{
* Corresponding authors. E-mail: tanchengxia@zjut.edu.cn; gfzhang@zjut.edu.cn Received September 5, 2018; revised October 22, 2018; published online November 25, 2018. Project supported by the Collaborative Innovation Center of Zhejiang Province Green Pesticide. 浙江省绿色农药 2011 协同创新中心开放基金资助项目.
} 


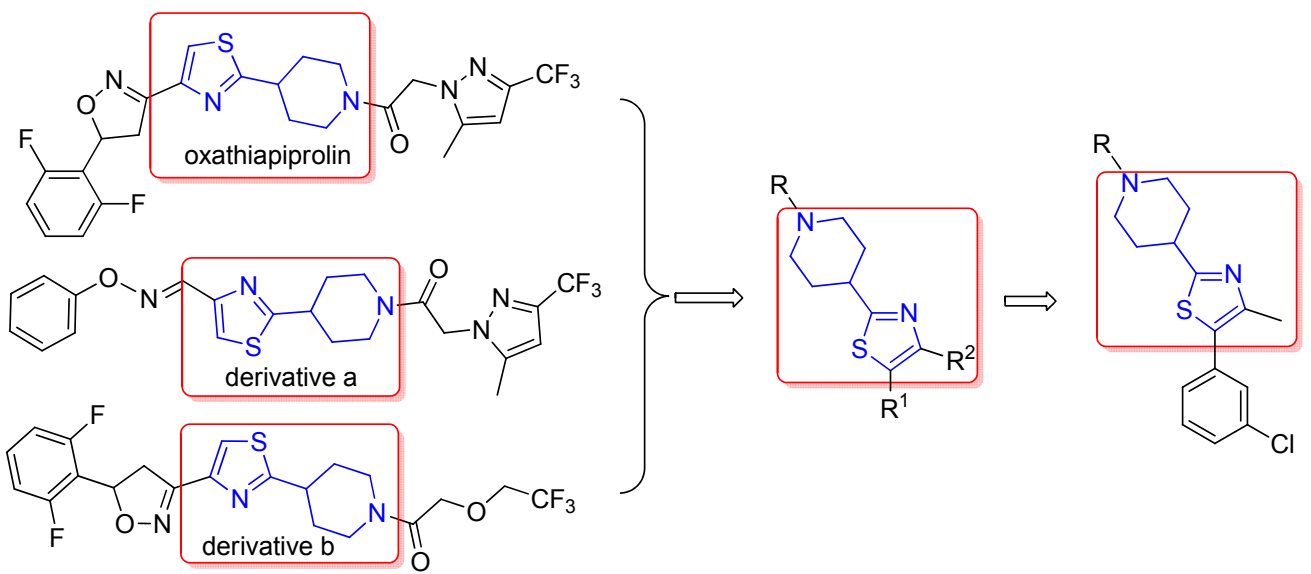

图 1 先导化合物设计

Figure 1 Design strategy of lead compound
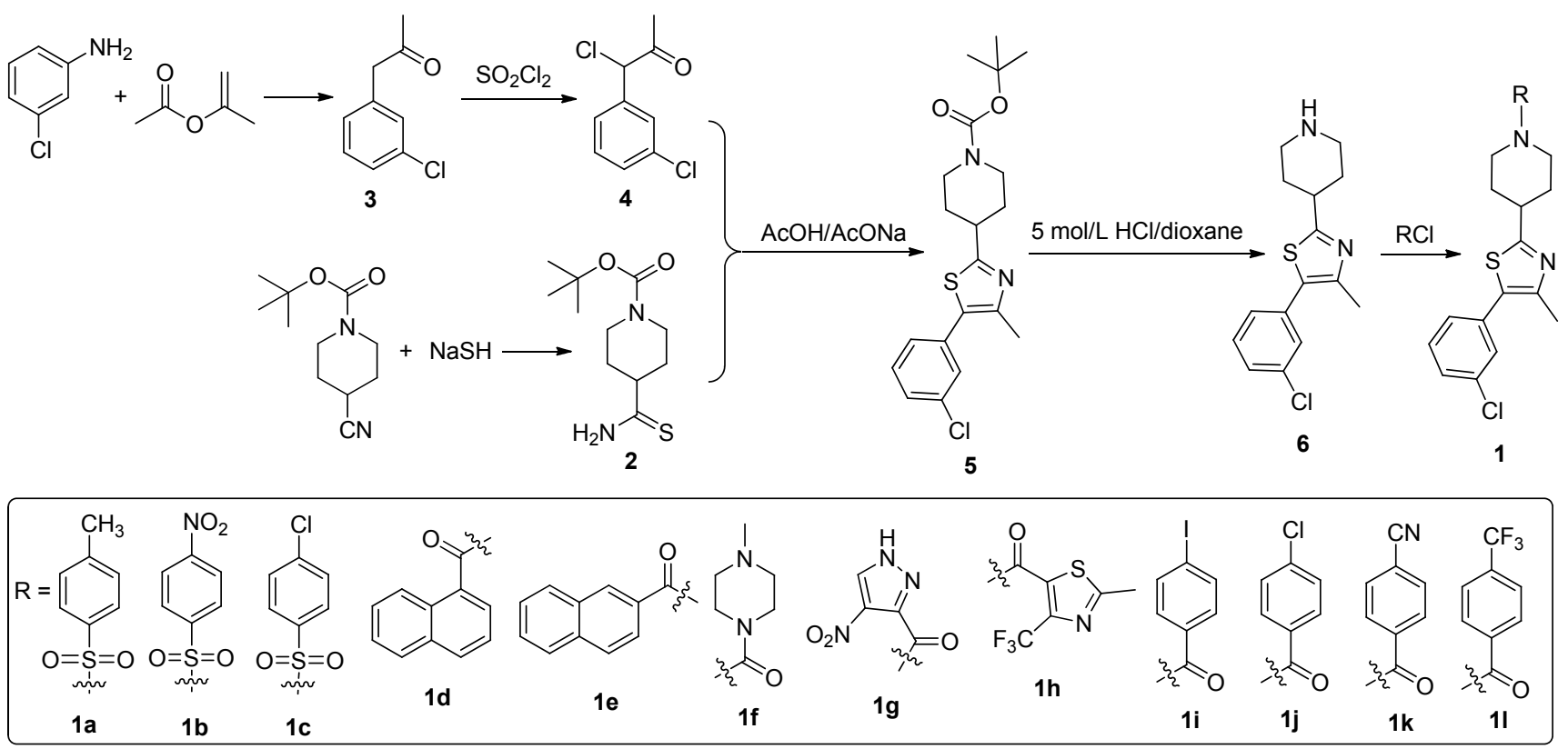

图式 1 目标化合物的合成路线

Scheme 1 Synthetic route of target compounds

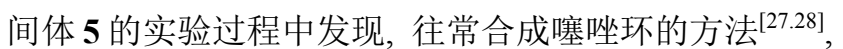
转化率基本在 $20 \%$ 左右, 有大量的原料剩余, 现只有在 冰醋酸作溶剂, 无水醋酸钠为缚酸剂, $80 \sim 85{ }^{\circ} \mathrm{C}$ 条件 下, 才能完全转化得到中间体 $\mathbf{5}^{[29]}$, 同时在实验中发现, 如果继续升温中间体 5 会直接转化成中间体 $\mathbf{6}$.

以目标化合物 $1 \mathbf{a}$ 为例进行谱学分析. ${ }^{1} \mathrm{H}$ NMR 谱图 中 $\delta 8.33 \sim 7.16$ 为芳环上的质子信号, $\delta 3.86,2.88,2.50$, $2.14,1.93 \sim 1.81$ 为哌啶环上质子信号; $\delta 2.35$ 为甲基上 的质子信号; 在 HRMS 谱图中, 该化合物分子离子峰计 算值为 $[\mathrm{M}+\mathrm{H}]^{+}$447.0952, 测定值 $[\mathrm{M}+\mathrm{H}]^{+}$447.0962, 绝对误差在 0.003 以内.

\section{2 化合物的杀虫活性}

目标化合物 $1 \mathrm{a} \sim 11$ 对粘虫的杀虫活性数据列于表 1, 在 $500 \mu \mathrm{g} / \mathrm{mL}$ 浓度下，化合物 $\mathbf{1 a} \sim 11$ 对粘虫表现出良 好的杀虫活性，大多数化合物对粘虫的致死率到达 $70 \%$ 以上，其中化合物 1f、1g 对 $100 \mu \mathrm{g} / \mathrm{mL}$ 的浓度下对粘虫 的致死率达到 $80 \%$ 以上. 继续对目标化合物 1f、1g 进行 杀虫活性，数据列于表 2 , 在 $20 \mu \mathrm{g} / \mathrm{mL}$ 浓度下，化合物 $1 \mathrm{~g}$ 对粘虫仍具有 $50 \%$ 的杀虫活性. 从 $1 \mathrm{~d}$ 与 $1 \mathrm{e}$ 的比较可 以发现，取代基的位置对活性的影响是巨大的; 从 1f、

$1 \mathrm{~g} 、 1 \mathrm{~h} 、 1 \mathrm{j}$ 的比较可以发现，芳香杂环种类的变化对目 标化合物的生物活性产生明显的影响, 为探究和优化该 类化合物提供了思路. 
表 1 目标化合物 1a 11 的杀虫活性(死亡率/\%)

Table 1 Insecticidal activities (mortality/\%) of target compounds 1a $\sim \mathbf{1 I}$

\begin{tabular}{|c|c|c|c|c|c|c|}
\hline \multirow{2}{*}{ Compd. } & \multicolumn{2}{|c|}{ Armyworm } & \multicolumn{2}{|c|}{ Aphis medicaginis Koc } & \multicolumn{2}{|c|}{ Tetranychus cinnabarinus } \\
\hline & $500 \mu \mathrm{g} / \mathrm{mL}$ & $100 \mu \mathrm{g} / \mathrm{mL}$ & $500 \mu \mathrm{g} / \mathrm{mL}$ & $100 \mu \mathrm{g} / \mathrm{mL}$ & $500 \mu \mathrm{g} / \mathrm{mL}$ & $100 \mu \mathrm{g} / \mathrm{mL}$ \\
\hline 5 & 80 & 0 & 0 & 0 & 0 & 0 \\
\hline 6 & 80 & 0 & 0 & 0 & 0 & 0 \\
\hline $1 \mathbf{a}$ & 0 & 0 & 0 & 0 & 0 & 0 \\
\hline $1 b$ & 80 & 0 & 0 & 0 & 0 & 0 \\
\hline $1 \mathrm{c}$ & 60 & 0 & 0 & 0 & 0 & 0 \\
\hline 1d & 0 & 0 & 0 & 0 & 0 & 0 \\
\hline $1 e$ & 80 & 0 & 0 & 0 & 0 & 0 \\
\hline 1f & 100 & 80 & 0 & 0 & 0 & 0 \\
\hline $1 \mathrm{~g}$ & 100 & 100 & 0 & 0 & 0 & 0 \\
\hline $1 \mathrm{~h}$ & 70 & 0 & 0 & 0 & 0 & 0 \\
\hline $\mathbf{1 i}$ & 80 & 0 & 0 & 0 & 0 & 0 \\
\hline $1 \mathrm{j}$ & 100 & 0 & 0 & 0 & 0 & 0 \\
\hline $1 \mathrm{k}$ & 70 & 0 & 0 & 0 & 0 & 0 \\
\hline 11 & 70 & 0 & 0 & 0 & 0 & 0 \\
\hline Abamectin & 100 & 100 & 100 & 100 & 100 & 100 \\
\hline
\end{tabular}

表 2 进一步篎选 $1 \mathrm{f}$ 和 $1 \mathrm{~g}$ 的杀虫活性(死亡率/\%)

Table 2 Further screening for insecticidal activities (mortality $/ \%$ ) of target compound $\mathbf{1 f}$ and $\mathbf{1 g}$

\begin{tabular}{ccc}
\hline \multirow{2}{*}{ Compd. } & \multicolumn{2}{c}{ Armyworm } \\
\cline { 2 - 3 } & $20 \mu \mathrm{g} / \mathrm{mL}$ & $4 \mu \mathrm{g} / \mathrm{mL}$ \\
\hline $\mathbf{1 f}$ & 0 & 0 \\
$\mathbf{1 g}$ & 50 & 0 \\
Abamectin & 100 & 100 \\
\hline
\end{tabular}

\section{2 结论}

以化合物 6 为结构骨架，设计并合成了 12 个含哌啶 噻唑类化合物, 结构经 ${ }^{1} \mathrm{H} N \mathrm{NMR},{ }^{13} \mathrm{C}$ NMR, HRMS 确认, 初步的生物活性测试结果表明, 部分目标化合物对粘虫 表现出较好的杀虫活性, 其中 $1 \mathrm{~g}$ 在 $100 \mu \mathrm{g} / \mathrm{mL}$ 浓度下对 粘虫具有 $100 \%$ 的杀虫活性, 在 $20 \mu \mathrm{g} / \mathrm{mL}$ 浓度下对粘虫 杀虫活性仍有 $50 \%$. 对于开发一种新型的哌啶噻唑类杀 虫剂具有很重要的研究意义和参考价值, 进一步的结构 优化和构效关系仍在进行中.

\section{3 实验部分}

\section{1 仪器与试剂}

质谱采用 Bruker Daltonics Bio-TOF-Q III 型质谱仪 (ESIMS) 测定; 天津光学仪器厂 Ry-1G 熔点仪, 温度计 未经校正; 核磁共振波谱使用 Bruker Avance 300 型核磁 共振仪测定. 所有试剂为国产市售分析纯.

\section{2 实验方法}

\subsubsection{N-boc-4-硫代酰胺哌啶(2)的合成}

在带机械摚拌的 $250 \mathrm{~mL}$ 的四口烧瓶中加入 $70 \%$ $\mathrm{NaSH}(14.2 \mathrm{~g}, 0.18 \mathrm{~mol}), \mathrm{NH}_{4} \mathrm{Cl}(10.0 \mathrm{~g}, 0.18 \mathrm{~mol}), N, N-$ 二甲基甲酰胺(DMF，120 mL)，4-氭基哌啶-1-甲酸叔丁
酯 $(8.6 \mathrm{~g}, 0.04 \mathrm{~mol})$, 在室温下搅拌 $72 \mathrm{~h}$, 反应完全后, 在 $1 \mathrm{~L}$ 的烧杯中加入 $600 \mathrm{~g}$ 冰水, 在搅拌过程中将 250 $\mathrm{mL}$ 四口瓶中的料液慢慢加入到冰水中, 搅拌 $1 \mathrm{~h}$ 后, 出 现大量白色固体，抽滤，滤液用乙酸乙酯萃取(100 $\mathrm{mL} \times 3)$, 旋蒸，与滤饼合并烘干，得中间体 2 , 收率 $95.2 \%$.

3.2.2 1-(间氯苯基)-2-丙酮(3)和 1-(间氯苯基)-2-氯丙酮(4)的合成

中间体 3 和中间体 4 分别参照文献[30]和[31]方法 合成.

3.2.3 4-(4-(3-氯苯基)-5-甲基噻唑-2-基)哌啶-1-甲酸 叔丁酯(5)的合成

在 $250 \mathrm{~mL}$ 三口瓶中加入中间体 $2(10.0 \mathrm{~g}, 0.05 \mathrm{~mol})$ 和化合物 4 (12.2 g, $0.05 \mathrm{~mol}), 100 \mathrm{~mL}$ 冰醋酸, $20 \mathrm{~g}$ 无水 醋酸钠, 在 $85{ }^{\circ} \mathrm{C}$ 搅拌条件下, 反应过夜, 用饱和 $\mathrm{NaHCO}_{3}$ 的水溶液调 $\mathrm{pH}=8 \sim 9$, 用乙酸乙酯萃取( 30 $\mathrm{mL} \times 3)$, 旋蒸, 经纯化得到中间体 $\mathbf{5}$, 收率 $72.4 \%$. 白色 固体, m.p. $145 \sim 147{ }^{\circ} \mathrm{C} ;{ }^{1} \mathrm{H}$ NMR $\left(500 \mathrm{MHz}, \mathrm{CDCl}_{3}\right) \delta$ : $7.44 \sim 7.31(\mathrm{~m}, 4 \mathrm{H}), 4.22(\mathrm{~s}, 2 \mathrm{H}), 3.13(\mathrm{tt}, J=11.7,3.7 \mathrm{~Hz}$, $1 \mathrm{H}), 2.88(\mathrm{~s}, 2 \mathrm{H}), 2.46(\mathrm{~s}, 3 \mathrm{H}), 2.20 \sim 2.04(\mathrm{~m}, 2 \mathrm{H}), 1.74$ (qd, $J=12.2,4.3 \mathrm{~Hz}, 2 \mathrm{H}), 1.48$ (s, 9H); ${ }^{13} \mathrm{C}$ NMR (126 $\left.\mathrm{MHz}, \mathrm{CDCl}_{3}\right) \delta: 172.26,154.71,147.35,133.73,130.72$, 130.37 (2C), 129.64, 128.90 (2C), 79.65, 43.71, 40.81 (2C), 32.47 (2C), 28.477(3C), 16.04. HRMS (ESI) calcd for $\mathrm{C}_{20} \mathrm{H}_{25} \mathrm{ClN}_{2} \mathrm{O}_{2} \mathrm{SNa}[\mathrm{M}+\mathrm{Na}]^{+} 415.1395$, found 415.1403.

3.2.4 4-(4-(3-氯苯基)-5-甲基噻唑-2-基)哌啶-1-盐酸 盐 $(6)$ 的合成

在 $100 \mathrm{~mL}$ 三口瓶中加入化合物 5 (10.0 g, 255 
$\mathrm{mmol}$ ), $5 \mathrm{~mol} / \mathrm{L} \mathrm{HCl}$ 二氧六环溶液 $20 \mathrm{~mL}$, 在室温下反 应 $4 \mathrm{~h}$, 减压抽滤, 得到中间体 6 , 收率为 $88.6 \%$. 白色固 体, m.p. $126 \sim 128{ }^{\circ} \mathrm{C} ;{ }^{1} \mathrm{H}$ NMR (500 MHz, DMSO- $d_{6}$ ) $\delta$ : $9.38(\mathrm{~d}, J=9.0 \mathrm{~Hz}, 1 \mathrm{H}), 9.24(\mathrm{~d}, J=8.8 \mathrm{~Hz}, 1 \mathrm{H}), 7.56 \sim$ $7.46(\mathrm{~m}, 4 \mathrm{H}), 3.41 \sim 3.25(\mathrm{~m}, 3 \mathrm{H}), 3.07 \sim 2.95(\mathrm{~m}, 2 \mathrm{H})$, $2.40(\mathrm{~s}, 3 \mathrm{H}), 2.22 \sim 2.13(\mathrm{~m}, 2 \mathrm{H}), 2.05 \sim 1.90(\mathrm{~m}, 2 \mathrm{H}) ;{ }^{13} \mathrm{C}$ NMR (126 MHz, DMSO- $d_{6}$ ) $\delta: 168.23,151.71,134.32$, $134.09,131.81,131.25,130.04,129.72,128.72,43.17$ (2C), 37.00, 29.00 (2C), 17.35. HRMS (ESI) calcd for $\mathrm{C}_{15} \mathrm{H}_{18} \mathrm{ClN}_{2} \mathrm{~S}[\mathrm{M}+\mathrm{H}]^{+}$293.0874, found 293.0882.

\section{2 .5 目标化合物 $1 \mathrm{a} \sim 1 \mathrm{1}$ 的合成}

在封管中加入中间体 $\mathbf{6}(0.10 \mathrm{~g}, 0.26 \mathrm{mmol})$ 、三乙胺 $(0.10 \mathrm{~g}, 0.99 \mathrm{mmol}), 1 \mathrm{~mL}$ 二氯甲烷和不同的酰氯或磺酰 氯, 反应 $2 \mathrm{~h}$, 水洗, 酸洗后, 用二氯甲烷萃取 $(10 \mathrm{~mL} \times$ $3)$, 旋蒸, 得到目标化合物 $\mathbf{1 a} \sim 1$ l.

5-(3-氯苯基)-4-甲基-2-(1-甲苯磺酰基哌啶-4-基)噻 唑(1a): 收率 90\%. 白色固体, m.p. 197 199 ${ }^{\circ} \mathrm{C} ;{ }^{1} \mathrm{H}$ NMR $\left(500 \mathrm{MHz}, \mathrm{CDCl}_{3}\right) \delta: 7.70 \sim 7.62(\mathrm{~m}, 2 \mathrm{H}), 7.41 \sim$ $7.30(\mathrm{~m}, 5 \mathrm{H}), 7.30 \sim 7.24(\mathrm{~m}, 1 \mathrm{H}), 3.89(\mathrm{~d}, J=12.5 \mathrm{~Hz}$, $2 \mathrm{H}), 2.91(\mathrm{tt}, J=11.7,3.8 \mathrm{~Hz}, 1 \mathrm{H}), 2.48 \sim 2.38(\mathrm{~m}, 8 \mathrm{H})$, $2.24 \sim 2.12(\mathrm{~m}, 2 \mathrm{H}), 1.92(\mathrm{dtd}, J=13.4,11.8,4.1 \mathrm{~Hz}, 2 \mathrm{H})$; ${ }^{13} \mathrm{C}$ NMR (126 MHz, $\left.\mathrm{CDCl}_{3}\right) \delta: 171.50,147.61,143.62$, $134.52,133.76,133.15,129.90,129.69$ (2C), 129.57, 129.01, 127.84, 127.65 (2C), 127.24, 45.93 (2C), 39.72, 31.78 (2C), 21.50, 15.95; HRMS (ESI) calcd for $\mathrm{C}_{22} \mathrm{H}_{24} \mathrm{ClN}_{2} \mathrm{O}_{2} \mathrm{~S}_{2}[\mathrm{M}+\mathrm{H}]^{+}$447.0952, found 447.0962.

5-(3-氯苯基)-4-甲基-2-(1-((4-硝基苯基)磺酰基)哌 啶-4-基)噻唑(1b): 收率 92\%. 白色固体, m.p. 210 $211{ }^{\circ} \mathrm{C} ;{ }^{1} \mathrm{H}$ NMR $\left(500 \mathrm{MHz}, \mathrm{CDCl}_{3}\right) \delta: 8.33(\mathrm{~d}, J=8.7$ $\mathrm{Hz}, 2 \mathrm{H}), 7.91(\mathrm{~d}, J=8.6 \mathrm{~Hz}, 2 \mathrm{H}), 7.34 \sim 7.21(\mathrm{~m}, 3 \mathrm{H})$, $7.21 \sim 7.16(\mathrm{~m}, 1 \mathrm{H}), 3.86(\mathrm{~d}, J=11.8 \mathrm{~Hz}, 2 \mathrm{H}), 2.88(\mathrm{t}, J=$ $11.6 \mathrm{~Hz}, 1 \mathrm{H}), 2.50$ (t, $J=11.6 \mathrm{~Hz}, 2 \mathrm{H}), 2.35$ (s, 3H), 2.14 $(\mathrm{d}, J=12.8 \mathrm{~Hz}, 2 \mathrm{H}), 1.93 \sim 1.81(\mathrm{~m}, 2 \mathrm{H}) ;{ }^{13} \mathrm{C}$ NMR $(126$ $\left.\mathrm{MHz}, \mathrm{CDCl}_{3}\right) \delta: 170.68,150.15,147.82,142.53,134.50$, $133.69,129.90,129.65,128.99,128.70$ (2C), 127.84, 127.22, 124.38 (2C), 45.82 (2C), 39.41, 31.60 (2C), 16.01; HRMS (ESI) calcd for $\mathrm{C}_{21} \mathrm{H}_{20} \mathrm{ClN}_{3} \mathrm{O}_{4} \mathrm{~S}_{2} \mathrm{Na}[\mathrm{M}+\mathrm{Na}]^{+}$ 500.0479 , found 500.0476 .

5-(3-氯苯基)-2-(1-((4-氯苯基)磺酰基)哌啶-4-基)-4甲基噻唑(1c): 收率 $95 \%$, 白色固体, m.p. $178 \sim 180{ }^{\circ} \mathrm{C}$; ${ }^{1} \mathrm{H}$ NMR $\left(500 \mathrm{MHz}, \mathrm{CDCl}_{3}\right) \delta: 7.73(\mathrm{~d}, J=9.2 \mathrm{~Hz}, 2 \mathrm{H})$, $7.54(\mathrm{~d}, J=8.4 \mathrm{~Hz}, 2 \mathrm{H}), 7.41 \sim 7.32(\mathrm{~m}, 3 \mathrm{H}), 7.32 \sim 7.25$ (m, 1H), $3.90(\mathrm{~d}, J=11.6 \mathrm{~Hz}, 2 \mathrm{H}), 3.00$ (tt, $J=11.7,3.9$ $\mathrm{Hz}, 1 \mathrm{H}), 2.55 \sim 2.41(\mathrm{~m}, 5 \mathrm{H}), 2.22(\mathrm{~d}, J=13.8 \mathrm{~Hz}, 2 \mathrm{H})$, $1.93(\mathrm{qd}, J=12.5,4.1 \mathrm{~Hz}, 2 \mathrm{H}) ;{ }^{13} \mathrm{C}$ NMR $(126 \mathrm{MHz}$,
$\left.\mathrm{CDCl}_{3}\right) \delta: 171.51,147.33,139.43,134.84,134.63,133.47$, 129.97, 129.80, 129.45 (2C), 129.04, 129.01 (2C), 128.04, 127.26, 45.85 (2C), 39.44, 31.70 (2C), 15.81; HRMS (ESI) calcd for $\mathrm{C}_{21} \mathrm{H}_{20} \mathrm{Cl}_{2} \mathrm{~N}_{2} \mathrm{O}_{2} \mathrm{~S}_{2} \mathrm{Na}[\mathrm{M}+\mathrm{Na}]^{+} 489.025$, found 489.0235 .

4-甲基-5-(3-氯苯基)-2-( $\alpha$-萫甲酰哌啶基)噻唑(1d): 收率 90\%, 白色固体, m.p. 197 199 ${ }^{\circ} \mathrm{C} ;{ }^{1} \mathrm{H}$ NMR (500 $\left.\mathrm{MHz}, \mathrm{CDCl}_{3}\right) \delta: 7.98 \sim 7.76(\mathrm{~m}, 3 \mathrm{H}), 7.62 \sim 7.35(\mathrm{~m}, 6 \mathrm{H})$, $7.28(\mathrm{dd}, J=8.5,2.4 \mathrm{~Hz}, 2 \mathrm{H}), 5.04(\mathrm{dt}, J=8.5,4.3 \mathrm{~Hz}$, $1 \mathrm{H}), 3.55(\mathrm{td}, J=9.0,8.2,3.8 \mathrm{~Hz}, 1 \mathrm{H}), 3.33(\mathrm{q}, J=11.3$ $\mathrm{Hz}, 1 \mathrm{H}), 3.22 \sim 3.01(\mathrm{~m}, 2 \mathrm{H}), 2.47(\mathrm{~d}, J=4.8 \mathrm{~Hz}, 4 \mathrm{H})$, $2.13 \sim 1.85(\mathrm{~m}, 2 \mathrm{H}), 1.68(\mathrm{dtd}, J=59.2,12.0,7.8 \mathrm{~Hz}, 1 \mathrm{H})$; ${ }^{13} \mathrm{C}$ NMR (126 MHz, $\left.\mathrm{CDCl}_{3}\right) \delta: 171.83,169.43,146.91$, $134.20,133.41,131.92$ (2C), 130.60 (2C), 130.02, 129.65, $129.46,129.10,128.41,127.03,126.43,125.17,124.71$, 123.62, 122.16, 47.08, 41.47, 40.32, 33.28, 32.30, 15.73; HRMS (ESI) calcd for $\mathrm{C}_{26} \mathrm{H}_{23} \mathrm{ClN}_{2} \mathrm{OSNa}[\mathrm{M}+\mathrm{Na}]^{+}$ 469.1128, found 469.1112 .

4-甲基-5-(间氯苯基)-2-( $\beta$-荎甲酰哌啶基)噻唑(1e): 收率 $92 \%$, 白色固体, m.p. $193 \sim 195^{\circ} \mathrm{C} ;{ }^{1} \mathrm{H}$ NMR $(500$ $\left.\mathrm{MHz}, \mathrm{CDCl}_{3}\right) \delta: 7.96 \sim 7.64(\mathrm{~m}, 4 \mathrm{H}), 7.58 \sim 7.02(\mathrm{~m}, 7 \mathrm{H})$, $4.78(\mathrm{~s}, 1 \mathrm{H}), 3.87(\mathrm{~s}, 1 \mathrm{H}), 3.31 \sim 2.78(\mathrm{~m}, 3 \mathrm{H}), 2.38(\mathrm{~s}$, $3 \mathrm{H}), 2.31 \sim 1.59(\mathrm{~m}, 4 \mathrm{H}) ;{ }^{13} \mathrm{C}$ NMR $\left(126 \mathrm{MHz}, \mathrm{CDCl}_{3}\right) \delta$ : $171.76,170.53,147.73,134.57,133.83,133.68,133.22$, $132.73,129.96,129.57,129.09,128.42,128.38,127.89$, 127.81, 127.31, 127.09, 126.74 (2C), 124.21, 47.60, 42.17, 40.66, 32.82, 32.41, 16.06; HRMS (ESI) calcd for $\mathrm{C}_{26} \mathrm{H}_{23} \mathrm{ClN}_{2} \mathrm{OSNa}[\mathrm{M}+\mathrm{Na}]^{+}$469.1128, found 469.1112.

4-甲基-5-(间氯苯基)-2-(4-甲基哌嗪-1-甲酰哌啶 基)噻唑(1f): 收率 93\%, 黄色油状物; ${ }^{1} \mathrm{H}$ NMR (500 $\left.\mathrm{MHz}, \mathrm{CDCl}_{3}\right) \delta: 7.37(\mathrm{t}, J=1.6 \mathrm{~Hz}, 1 \mathrm{H}), 7.35 \sim 7.23(\mathrm{~m}$, $3 \mathrm{H}), 3.78(\mathrm{~d}, J=13.3 \mathrm{~Hz}, 2 \mathrm{H}), 3.36 \sim 3.22(\mathrm{~m}, 4 \mathrm{H}), 3.14 \sim$ $3.05(\mathrm{~m}, 1 \mathrm{H}), 2.94 \sim 2.85(\mathrm{~m}, 2 \mathrm{H}), 2.48 \sim 2.35(\mathrm{~m}, 7 \mathrm{H})$, 2.29 (d, $J=4.4 \mathrm{~Hz}, 3 \mathrm{H}), 2.10(\mathrm{~d}, J=10.7 \mathrm{~Hz}, 2 \mathrm{H}), 1.79$ (qd, $J=12.5,3.9 \mathrm{~Hz}, 2 \mathrm{H}) ;{ }^{13} \mathrm{C} \mathrm{NMR}\left(126 \mathrm{MHz}, \mathrm{CDCl}_{3}\right) \delta$ : 172.20, 163.89, 147.74, 134.48, 134.05, 129.86, 129.29, 129.04, 127.69, 127.25, 54.74 (2C), 46.84 (2C), 46.62 (2C), 46.07, 40.93, 32.35 (2C), 16.11; HRMS (ESI) calcd for $\mathrm{C}_{21} \mathrm{H}_{28} \mathrm{ClN}_{4} \mathrm{OS}[\mathrm{M}+\mathrm{H}]^{+} 419.1659$, found 419.1667 .

(4-(5-(3-氯苯基)-4-甲基噻唑-2-基)哌啶-1-基)(4-硝 基- $1 H$-吡唑-3-基)甲酮(1g): 收率 93\%, 褐色油状物; ${ }^{1} \mathrm{H}$ NMR (500 MHz, DMSO- $\left.d_{6}\right) \delta: 8.87(\mathrm{~s}, 1 \mathrm{H}), 7.52 \sim 7.37$ (m, 4H), 4.59 (d, $J=12.2 \mathrm{~Hz}, 1 \mathrm{H}), 3.50(\mathrm{~d}, J=15.5 \mathrm{~Hz}$, $1 \mathrm{H}), 3.31$ (tq, $J=10.9,3.5 \mathrm{~Hz}, 1 \mathrm{H}), 3.26 \sim 3.16(\mathrm{~m}, 1 \mathrm{H})$, $3.09 \sim 2.99(\mathrm{~m}, 2 \mathrm{H}), 2.39(\mathrm{~d}, J=2.2 \mathrm{~Hz}, 3 \mathrm{H}), 2.22 \sim 2.10$ 
(m, 1H), $1.99(\mathrm{~d}, J=14.0 \mathrm{~Hz}, 1 \mathrm{H}), 1.63(\mathrm{dqd}, J=41.0$, 12.2, 4.3 Hz, 2H); ${ }^{13} \mathrm{C}$ NMR (126 MHz, DMSO- $\left.d_{6}\right) \delta$ : $171.26,160.03,147.60,141.50,133.70,133.51,131.78$, $131.15,130.70,128.56,128.23,127.67,127.54,45.86$, $45.58,40.84,32.21,31.56,16.01$; HRMS (ESI) calcd for $\mathrm{C}_{19} \mathrm{H}_{18} \mathrm{ClN}_{5} \mathrm{O}_{3} \mathrm{SNa}[\mathrm{M}+\mathrm{Na}]^{+}$454.0700, found 454.0711.

(4-(5-(3-氯苯基)-4-甲基噻唑-2-基)哌啶-1-基)-(4-甲 基-2-(三氟甲基)噻唑-5-基)甲酮(1h)：收率 91\%, 白色固 体, m.p. $176 \sim 178{ }^{\circ} \mathrm{C} ;{ }^{1} \mathrm{H}$ NMR $\left(500 \mathrm{MHz}, \mathrm{CDCl}_{3}\right) \delta$ : $7.45 \sim 7.25(\mathrm{~m}, 4 \mathrm{H}), 4.75(\mathrm{~d}, J=12.3 \mathrm{~Hz}, 1 \mathrm{H}), 3.69$ (d, $J=$ $13.1 \mathrm{~Hz}, 1 \mathrm{H}), 3.26(\mathrm{dd}, J=22.8,10.6 \mathrm{~Hz}, 2 \mathrm{H}), 3.05(\mathrm{t}, J=$ $11.8 \mathrm{~Hz}, 1 \mathrm{H}), 2.76$ (s, 3H), 2.47 (s, 3H), 2.26 (d, $J=12.0$ $\mathrm{Hz}, 1 \mathrm{H}), 2.15(\mathrm{~d}, J=11.9 \mathrm{~Hz}, 1 \mathrm{H}), 1.95 \sim 1.70(\mathrm{~m}, 2 \mathrm{H})$; ${ }^{13} \mathrm{C}$ NMR (126 MHz, $\left.\mathrm{CDCl}_{3}\right) \delta: 171.10,167.72 .159 .11$, $147.66,139.44$ (q, $J=36.6 \mathrm{~Hz}), 134.59,133.62,131.98$, $129.94,129.76,129.06,127.96,127.28,120.20$ (q, $J=$ $271.7 \mathrm{~Hz}), 47.13$, 41.99, 40.10, 32.21, 31.68, 19.08, 15.96; HRMS (ESI) calcd for $\mathrm{C}_{21} \mathrm{H}_{19} \mathrm{ClF}_{3} \mathrm{~N}_{3} \mathrm{OS}_{2} \mathrm{Na}[\mathrm{M}+\mathrm{Na}]^{+}$ 508.0522 , found 508.0502 .

(4-(5-(3-氯苯基)-4-甲基噻唑-2-基)哌啶-1-基)(4-碘 苯基)甲酮(1i): 收率 $88 \%$, 白色固体, m.p. $176 \sim 178{ }^{\circ} \mathrm{C}$; ${ }^{1} \mathrm{H}$ NMR $\left(500 \mathrm{MHz}, \mathrm{CDCl}_{3}\right) \delta: 7.77 \sim 7.69(\mathrm{~m}, 2 \mathrm{H})$, $7.57 \sim 7.49(\mathrm{~m}, 2 \mathrm{H}), 7.41 \sim 7.32(\mathrm{~m}, 3 \mathrm{H}), 7.28(\mathrm{dt}, J=6.9$, $1.8 \mathrm{~Hz}, 1 \mathrm{H}), 3.91(\mathrm{dt}, J=12.7,3.8 \mathrm{~Hz}, 2 \mathrm{H}), 3.13 \sim 3.04$ (m, $1 \mathrm{H}), 2.54 \sim 2.44(\mathrm{~m}, 5 \mathrm{H}), 2.24(\mathrm{dt}, J=13.4,2.3 \mathrm{~Hz}$, $2 \mathrm{H}), 1.99 \sim 1.87(\mathrm{~m}, 2 \mathrm{H}) ;{ }^{13} \mathrm{C} \mathrm{NMR}\left(126 \mathrm{MHz}, \mathrm{CDCl}_{3}\right) \delta$ : $172.07,146.67,139.51,134.75,133.02,130.09,129.59$, 129.49 (2C), 129.41, 129.06, 129.02 (2C), 128.32, 128.27, 127.27, 45.84 (2C), 39.21, 31.73 (2C), 15.55; HRMS (ESI) calcd for $\mathrm{C}_{22} \mathrm{H}_{20} \mathrm{ClIN}_{2} \mathrm{OSNa}[\mathrm{M}+\mathrm{Na}]^{+}$544.9901, found 544.9922.

(4-氯苯基)-(4-(5-(3-氯苯基)-4-甲基噻唑-2-基)哌啶1-基)甲酮(1j): 收率 $86 \%$, 白色固体, m.p. $174 \sim 176^{\circ} \mathrm{C}$; ${ }^{1} \mathrm{H}$ NMR $\left(500 \mathrm{MHz}, \mathrm{CDCl}_{3}\right) \delta: 7.44 \sim 7.32(\mathrm{~m}, 7 \mathrm{H}), 7.30$ (dt, $J=7.2,1.7 \mathrm{~Hz}, 1 \mathrm{H}), 4.78(\mathrm{~s}, 1 \mathrm{H}), 3.88(\mathrm{~s}, 1 \mathrm{H}), 3.36 \sim$ $2.87(\mathrm{~m}, 3 \mathrm{H}), 2.48(\mathrm{~s}, 3 \mathrm{H}), 2.25$ (s, 2H), 1.80 (d, $J=30.2$ $\mathrm{Hz}, 2 \mathrm{H}) ;{ }^{13} \mathrm{C}$ NMR $\left(126 \mathrm{MHz}, \mathrm{CDCl}_{3}\right) \delta: 171.50,169.40$, $147.81,135.80,134.60,134.30,133.84,129.94,129.58$ (2C), 129.11, 128.80, 128.48 (2C), 127.89, 127.30, 47.54, 42.24, 40.58, 32.46, 29.69, 16.06; HRMS (ESI) calcd for $\mathrm{C}_{22} \mathrm{H}_{20} \mathrm{Cl}_{2} \mathrm{~N}_{2} \mathrm{OSNa}[\mathrm{M}+\mathrm{Na}]^{+} 453.0571$, found 453.0566 .

((5-(3-氯苯基)-4-甲基噻唑-2-基)哌啶-1-羰基)苯甲 腈(1k): 收率 90\%, 褐色固体, m.p. 184 186 ${ }^{\circ} \mathrm{C} ;{ }^{1} \mathrm{H}$ NMR (500 MHz, $\left.\mathrm{CDCl}_{3}\right) \delta: 7.66(\mathrm{~d}, J=7.8 \mathrm{~Hz}, 2 \mathrm{H}), 7.47$ (d, $J=7.9 \mathrm{~Hz}, 2 \mathrm{H}), 7.38 \sim 7.07(\mathrm{~m}, 4 \mathrm{H}), 4.84 \sim 4.62(\mathrm{~m}$,
1H), $3.69(\mathrm{~d}, J=10.5 \mathrm{~Hz}, 1 \mathrm{H}), 3.46 \sim 3.30(\mathrm{~m}, 1 \mathrm{H}), 3.07$ (d, $J=17.3 \mathrm{~Hz}, 2 \mathrm{H}), 2.45$ (s, 3H), 2.23 (s, 2H); $1.97 \sim 1.64$ (m, 2H); ${ }^{13} \mathrm{C}$ NMR $\left(126 \mathrm{MHz}, \mathrm{CDCl}_{3}\right) \delta: 172.21,168.27$, 146.41, 140.09, 134.73, 132.42 (2C), 130.10 (2C), 129.01, 128.41 (2C), 127.58, 127.23 (2C), 117.97, 113.49, 47.26, 41.92, 39.81, 32.68, 31.90, 15.39; HRMS (ESI) calcd for $\mathrm{C}_{23} \mathrm{H}_{21} \mathrm{ClN}_{3} \mathrm{OS}[\mathrm{M}+\mathrm{H}]^{+} \quad 422.1072$, found 422.1088 .

4-甲基-5-(3-氯苯基)-2-(对三氟甲基苯甲酰哌啶基) 噻唑(11): 收率 91\%, 白色固体, m.p. 201 203 ${ }^{\circ} \mathrm{C} ;{ }^{1} \mathrm{H}$ NMR $\left(500 \mathrm{MHz}, \mathrm{CDCl}_{3}\right) \delta: 7.62(\mathrm{~d}, J=7.4 \mathrm{~Hz}, 2 \mathrm{H}), 7.55$ (d, $J=7.7 \mathrm{~Hz}, 1 \mathrm{H}), 7.49$ (t, $J=7.6 \mathrm{~Hz}, 1 \mathrm{H}), 7.31$ (q, $J=$ $7.9,6.9 \mathrm{~Hz}, 3 \mathrm{H}), 7.23(\mathrm{~s}, 1 \mathrm{H}), 4.86 \sim 4.63(\mathrm{~m}, 1 \mathrm{H}), 3.75(\mathrm{~s}$, $1 \mathrm{H}), 3.44$ (s, 1H), 3.00 (d, $J=37.0 \mathrm{~Hz}, 2 \mathrm{H}), 2.47$ (s, $3 \mathrm{H})$, $2.19(\mathrm{~d}, J=35.3 \mathrm{~Hz}, 2 \mathrm{H}), 1.78(\mathrm{~d}, J=58.4 \mathrm{~Hz}, 2 \mathrm{H}) ;{ }^{13} \mathrm{C}$ NMR $\left(126 \mathrm{MHz}, \mathrm{CDCl}_{3}\right) \delta: 145.87,136.47,134.80$, 132.38, 131.04 (q, $J=32.4 \mathrm{~Hz}$ ), 130.27, 130.17 (2C), $129.13,128.99,128.57,127.22,126.58 \sim 126.41$ (m, 2C), 123.90 (q, $J=3.8 \mathrm{~Hz}, 2 \mathrm{C}$ ), 123.56 (q, $J=272.5 \mathrm{~Hz}$ ), 47.42, 41.86, 39.67, 32.70, 31.89, 15.13; HRMS (ESI) calcd for $\mathrm{C}_{23} \mathrm{H}_{20} \mathrm{ClF}_{3} \mathrm{~N}_{2} \mathrm{OSNa}[\mathrm{M}+\mathrm{Na}]^{+} 487.084$, found 487.0829 .

\section{3 生物活性测试}

\subsection{1 目标化合物对粘虫的杀虫活性}

采用浸渍法测定目标化合物对蚜虫的杀虫活性. 对 于目标化合物用分析天平 $(0.0001 \mathrm{~g})$ 称取一定质量的原 药, 用含 $1 \%$ 吐温- 80 乳化剂的 DMF 溶解配制成 $1 \%$ 母液, 然后用蒸馏水稀释备用, 每个处理三个重复, 设空白对 照, 阿维菌素作为对照药剂.

将适量玉米叶在配好的药液中充分浸润后自然阴 干，放入垫有滤纸的培养皿中，接粘虫 3 龄中期幼虫 10 头/血, 置于 $24 \sim 27{ }^{\circ} \mathrm{C}$ 观察室内培养, $3 \mathrm{~d}$ 后调查结果. 以毛笔触动虫体, 无反应视为死虫, 并进行统计分析.

\subsection{2 目标化合物对苜宿蚜和朱砂叶螨的杀虫活性}

采用浸渍法测定目标化合物对苜葆蚜和朱砂叶螨 的杀虫活性 ${ }^{[32]}$. 对于目标化合物用分析天平 $(0.0001 \mathrm{~g})$ 称取一定质量的原药, 用含 $1 \%$ 吐温- 80 乳化剂的 DMF 溶解配制成 $1 \%$ 母液, 然后用蒸馏水稀释备用, 每个处 理三个重复, 设空白对照, 阿维菌素作为对照药剂.

分别将接有朱砂叶螨和苜葆蚜的䖯豆叶片于 Potter 喷雾塔下喷雾处理, 处理后朱砂叶螨置于 $24 \sim 27{ }^{\circ} \mathrm{C}$ 观 察室内培养, 苜葆蚜置于 $20 \sim 22{ }^{\circ} \mathrm{C}$ 观察室内培养, $48 \mathrm{~h}$ 后调查结果. 以毛笔触动虫体, 无反应视为死虫, 并进 行统计分析.

辅助材料(Supporting Information) 化合物 1a $\sim 11$ 的 ${ }^{1} \mathrm{H}$ NMR, ${ }^{13} \mathrm{C}$ NMR 和 HRMS 图谱. 这些材料可以免费 
从本刊网站(http://sioc-journal.cn/)上下载.

\section{References}

[1] Huang, G.; Yang, J. -C.; Li, H.-C. Agrochemicals 2011, 50, 79 (in Chinese.) (黄光，杨吉春，李慧超，农药，2011，50, 79.)

[2] Yang, Z.-H.; Tian, H.; Zhang, L. World Pestic. 2017, 39, 43 (in Chinese). (杨子辉，田昊，张莉，世界农药, 2017, 39, 43.)

[3] Tan, Y.; Zhang, B.-H. J. Math. Med. 2012, 25, 89 (in Chinese). (覃宇, 张宝徽, 数理医药学杂志, 2012, 25, 89.)

[4] Biller, S. A. US 5739135, 1998 [Chem. Abstr. 1998, 128, 282780].

[5] Guedat, P. US 20070054939, 2007 [Chem. Abstr. 2007, 142, 93809].

[6] Li, Y. CN 106588911, 2017 [Chem. Abstr. 2017, 166, 515724].

[7] Fan, Z. J. CN 104650060, 2015 [Chem. Abstr. 2015, 163, 65937].

[8] Gu, L.-L.; Bai, Y.-L. Mod. Agrochem. 2017, 16, 42 (in Chinese.) (顾林玲, 柏亚罗, 现代农药, 2017, 16, 42.)

[9] He, X.-L. World Pestic. 2015, 37, 57 (in Chinese). (何秀玲, 世界农药, 2015, 37, 57.)

[10] Cederbaum, F. WO 2014118142, 2014 [Chem. Abstr. 2014, 161, 320959].

[11] Lamberth, C. WO 2014154530, 2014 [Chem. Abstr. 2014, 161, 640429].

[12] Tomoki, T. US 20130296272, 2013 [Chem. Abstr. 2012, 156, 337332].

[13] Gregory, V. WO 2009055514, 2009 [Chem. Abstr. 2009, 150, 578441].

[14] Stefan, H. US 20160198713, 2016 [Chem. Abstr. 2016, 162, 400022].

[15] Pierre, C. US 20110312999, 2011 [Chem. Abstr. 2011, 155, 615369].

[16] Hanagan, M. A. WO 2009094407, 2009 [Chem. Abstr. 2009, 151, 234956].

[17] Kamireddy, B. WO 2009094445, 2009 [Chem. Abstr. 2009, 151,
173451]

[18] Pasteris, R. J. WO 2008013925, 2008 [Chem. Abstr. 2008, 148, 185163].

[19] Yi, A.-Q.; Xue, S.-J.; Fang, Z.-K. Chin. J. Org. Chem. 2009, 29, 454 (in Chinese).

(尹安琴, 薛思佳, 方治坤, 有机化学, 2009, 29, 454.)

[20] Hong, Y.; Dai, H.; Ye, Y.-L. Chin. J. Org. Chem. 2017, 37, 3006 (in Chinese).

(洪宇, 戴红, 叶林玉，有机化学, 2017, 37, 3006.)

[21] Gao, H.; Zheng, X.; Zhu, P. Chin. J. Org. Chem. 2018, 38, 684 (in Chinese).

(高慧, 郑喜, 朱萍, 有机化学, 2018, 38, 684.)

[22] Zhang, Z.-H.; Chen, Y.; Cai, B.-S. Chin. J. Org. Chem. 2017, 37, 2377 (in Chinese).

(张志华, 陈羽, 柴宝山, 有机化学, 2017, 37, 2377.)

[23] Cao, L.; Sun, J.-W.; Liu, Q. Chin. J. Org. Chem. 2017, 37, 3031 (in Chinese). (曹蕾, 孙景伟, 刘强, 有机化学, 2017, 37, 3031.)

[24] Ma, H.-L.; Yan, X.-J.; Xiao, Y.-M. Chin. J. Org. Chem. 2016, 36, 158 (in Chinese)

(麻红利, 闵晓静, 肖玉梅, 有机化学, 2016, 36, 158.)

[25] Sun, N.; Wang, X.; Ding, Z.-B. Chin. J. Org. Chem. 2016, 36, 2489 (in Chinese). (孙楠, 王欣, 丁志彬, 有机化学, 2016, 36, 2489.)

[26] Li, R.; Nakashima, T.; Galangau, O. Chem. Asian J. 2015, 10, 1725.

[27] Khillare, L. D.; Pratap, U. R.; Bhosle, M. R. Chem. Intermed. 2017, $12,1$.

[28] Tong, D.; Duan, H.; Wang, J. Chem. Res. Chin. Univ. 2014, 30, 4.

[29] Hantzsch, A.; Weber, J. H. Chem. Ber. 1887, 20, 3118.

[30] Parsons P. J.; Johnathan. B.; Waters A. J. Synth. Commun. 2007, 37, 985.

[31] Srivatsavati, Jagapathi, R.; Pothukuchi, S.; Rani, S.-S. WO 2012143933, 2012 [Chem. Abstr. 2012, 167, 481902].

[32] Dai, H.; Ye, L. Y.; Zhuang, H. Y.; Dai, B. J.; Fang, Y.; Shi, Y. J. Molecules 2015, 20, 21870.

(Cheng, F.) 